\title{
El diagnóstico diferencial es fundamental: a propósito de un caso de síndrome de Laugier-Hunziker
}

\author{
The differential diagnosis is crucial: a case of Laugier-Hunziker syndrome
}

\author{
María Dolores González-Vázquez¹, Marta Rafael-Marques ${ }^{1}$ \\ ${ }^{1}$ Departamento de Medicina Interna. Centro Hospitalar Póvoa de Varzim - Vila do Conde
}

\section{RESUMEN}

El síndrome de Laugier-Hunziker (SLH) es una enfermedad poco frecuente, benigna, no asociada a patología sistémica, cuya forma de presentación en adultos consiste en hiperpigmentación macular mucocutánea que afecta mayoritariamente a labios y mucosa oral, ocasionalmente puede afectar mucosa genital y con menos frecuencia al área palmoplantar. Se encuentra asociada en la mitad de los casos con melanoniquia longitudinal. La importancia de este síndrome es evidente una vez que es diagnóstico de exclusión de enfermedades con significativo riesgo de malignidad. Ante la sospecha de SLH, es obligatorio el despistaje de otras patologías asociadas a hiperpigmentación, lentiginosis y melanoniquia. Los autores presentan el caso de una mujer de 63 años con hiperpigmentación de mucosa oral y de dedos de manos y pies, con el diagnóstico final de SLH. Realizan un resumen del estudio realizado que condujo al diagnóstico. Concluyen subrayando la importancia del diagnóstico diferencial con otras patologías.

Palabras clave: mucosa oral, hiperpigmentación, Laugier-Hunziker, diagnóstico diferencial.

Keywords: oral mucosa, hyperpigmentation, Laugier-Hunziker, differential diagnosis.

\section{INTRODUCCIÓN}

El síndrome de Laugier-Hunziker (SLH) es una enfermedad poco frecuente, benigna, no asociada a patología sistémica, cuya forma de presentación en adultos consiste en hiperpigmentación macular mucocutánea que afecta mayoritariamente a labios y mucosa oral, ocasionalmente puede afectar mucosa genital y con menos frecuencia al área palmoplantar. Existe asociación de este síndrome con melanoniquia ungueal longitudinal.

El SLH se considera una enfermedad adquirida benigna que no se asocia a anomalías sistémicas subyacentes ni a factores predisponentes ${ }^{2,3,5}$. La importancia de esta patología se basa en su diagnóstico diferencial. Ante la sospecha de SLH es obligatorio realizar el diagnóstico de exclusión con otras enfermedades con mayor riesgo de malignidad, a través del despiste de todas las patologías asociadas a hiperpigmentación, lentiginosis y melanoniquia².

\section{CASO CLÍNICO}

Se presenta el caso de una mujer de 63 años, caucásica, no fumadora, seguida en consulta de Medicina Interna en nuestro hospital, derivada desde Atención Primaria por diabetes mellitus tipo 2 de difícil control metabólico con afectación sistémica, a destacar como antecedente patológico personal relevante, la hipertensión arterial.

En la primera consulta nos llamó la atención la existencia de unas lesiones hiperpigmentadas localizadas en mucosa oral (imagen 1) y en dedos de manos y pies (imagen 2 y 3). Según la paciente las primeras lesiones habían aparecido aproximadamente dos años antes, y refería un empeoramiento progresivo sobretodo en las lesiones de los labios y de los dedos de las manos, no presentaba ninguna sintomatología asociada.

Fue realizada una historia clínica detallada siendo excluida historia familiar de alteraciones cutáneas y otros anteceden- tes familiares relevantes. Tampoco existía historia previa de trauma en las áreas afectadas por las lesiones o toma de fármacos que pudieran causar los cambios descritos.

Fue realizado un examen físico exhaustivo, no fueron encontradas otras alteraciones excepto las ya referidas: existencia de múltiples lesiones lenticulares irregulares de color castaño y negro con dimensiones entre 3-7 $\mathrm{mm}$ de diámetro en los labios y mucosa oral, y de menor tamaño en dedos de manos y pies.

Se realizó un estudio analítico completo incluyendo estudio inmunológico, detección de posibles déficits vitamínicos y despistaje de insuficiencia suprarrenal. Todos los resultados obtenidos se encontraban dentro de los parámetros de normalidad.

Ante la sospecha de SLH fue solicitado apoyo de Dermatología para realización de biopsia de las lesiones. El resultado mostró presencia de hiperqueratosis, acantosis e hiperpigmentación de células basales con algunos melanocitos. Se llevó a cabo un estudio complementario extenso para exclusión de otras patologías de mayor potencial de malignidad, ecocardiograma que mostró alteraciones compatibles con cardiopatía hipertensiva, TC de tórax y abdomen que no presentó hallazgos patológicos, estudio digestivo con endoscopia alta y colonoscopia para exclusión de enfermedad gastrointestinal asociada, ambos sin ninguna alteración.

La presentación clínica indolente, la ausencia de hallazgos relevantes en el estudio diagnóstico realizado, junto con la histopatología de la biopsia de la piel condujeron a la confirmación diagnóstica de SLH. Actualmente, después de tres años de seguimiento conjunto en Medicina Interna y Dermatología, no han sido detectadas alteraciones sistémicas asociadas ni evolución para malignidad de las lesiones descritas. 
Figura 1

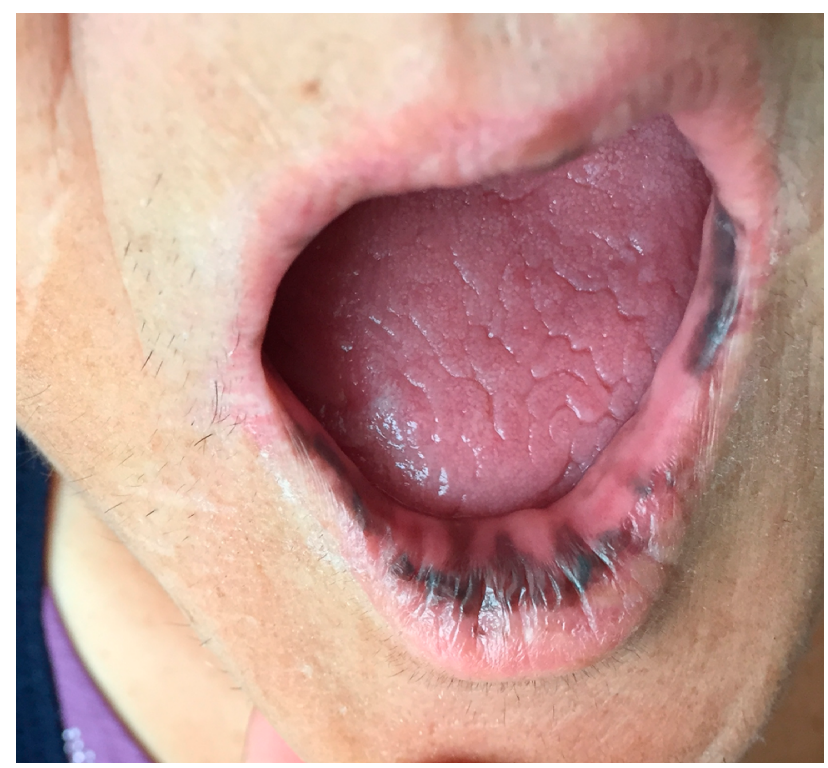

Figura 2

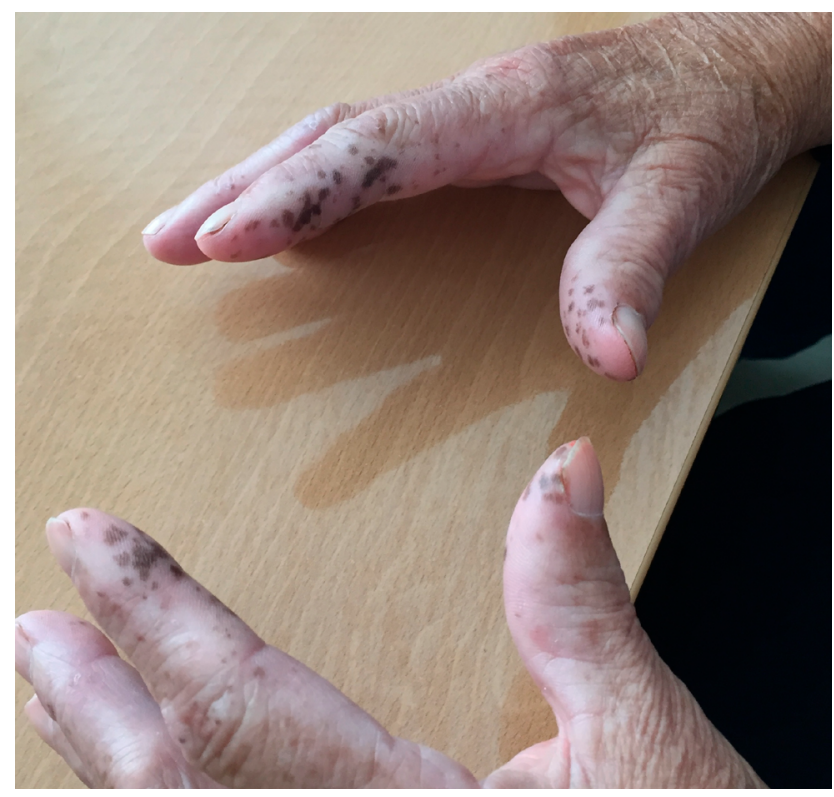

Figura 3

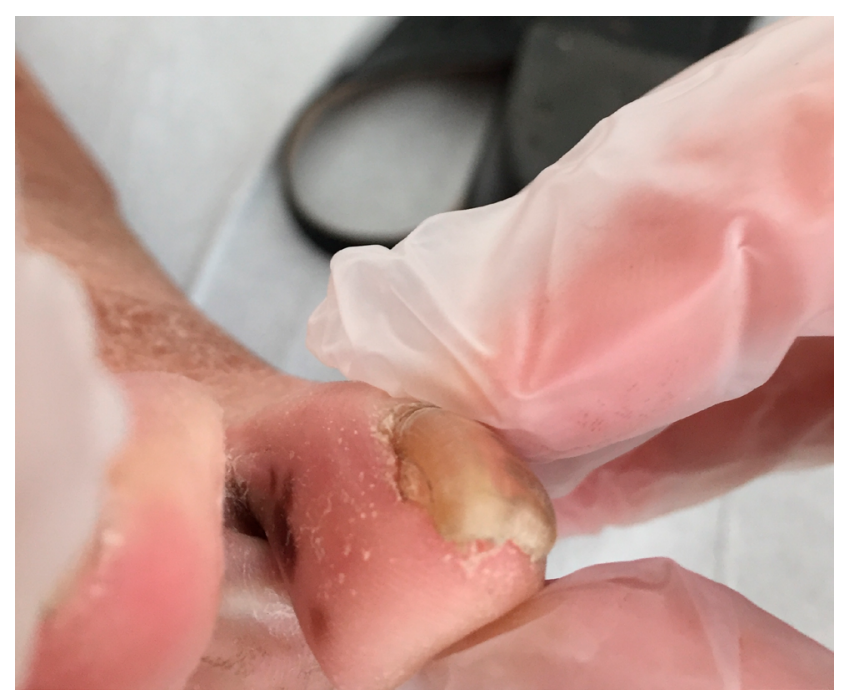

\section{COMENTARIO}

La primera descripción del fenómeno caracterizado por la aparición de pigmentación en mucosa oral y genital en adultos fue realizada por Laugier y Hunziker en 19701,4. Posteriormente, Baran ${ }^{5}$ relacionó dicho fenómeno con melanoniquia longitudinal y describió la pigmentación ungueal en el SLH, clasificándola en tres tipos. Posteriormente Veraldi et al ${ }^{2}$ reportó un cuarto tipo de melanoniquia.

Por su presentación benigna, no asociada a otras manifestaciones sistémicas, Moore et al. propusieron evitar el término "síndrome" para definir la alteración descrita por Laugier y Hunziker, considerando más apropiado la denominación de "pigmentación de Laugier-Hunziker"8,9

Desde ese momento y de acuerdo con una reciente revisión de la literatura existen aproximadamente 206 casos reportados (de los cuales dos fueron descritos en España y dos en Portugal). La edad media en el momento del diagnóstico es de 47,5 años, siendo más frecuente en mujeres con una ratio de 1,8:13. La etiología exacta permanece desconocida.

La manifestación clínica consiste en la presencia de máculas lenticulares melánicas en el área labial, la cavidad oral y regiones acrales, identificándose la melanoniquia longitudinal en aproximadamente mitad de los pacientes. En nuestro caso es importante observar la presencia de lesiones en los dedos de los pies ya que se trata de un área descrita como raramente afectada. Los hallazgos dermatoscópicos de las lesiones labiales incluyen presencia de un patrón de surco paralelo con múltiples puntos marrones y alteraciones reticulares regulares, múltiples patrones granulares de color marrón 0 gris, estrías en arco, similares a escamas de pescado y surcos paralelos en lesiones pigmentarias mucocutáneas. Estos patrones dermatoscópicos se observan frecuentemente junto con vasos lineales y punteados en áreas rosadas blanquecinas ${ }^{4}$. El signo de Pseudo-Hutchison puede aparecer cuando existen alteraciones a nivel ungueal ${ }^{3}$.

El examen histológico de las lesiones muestra aumento de los depósitos de melanina en las células basales, siendo que el número, morfología y distribución de melanocitos habitualmente es normal ${ }^{5}$. Con poca frecuencia, a semejanza con nuestro caso, se han reportado hallados como acantosis ${ }^{7}$, hiperqueratosis ${ }^{8}$ e hiperparaqueratosis 7 .

Siendo el diagnóstico de SLH un diagnóstico de exclusión, se obtiene después de la investigación de otras causas de pigmentación oral, labial y ungueal como por ejemplo: Síndrome de Peutz-Jeghers (SPJ), Síndrome de LEOPARD (es un acrónimo de las principales características de este trastorno, que incluye léntigos múltiples, anomalías en la conducción del electrocardiograma [ECG], hipertelorismo ocular, estenosis pulmonar, genitales anormales, retraso del crecimiento y sordera neurosensorial), Enfermedad de Addison, Síndrome de McCune-Albright ${ }^{10}$, Síndrome de LAMB o Complejo de Carney ${ }^{11}$ (léntigos en piel y mucosas, mixomas auriculares), 
Tabla 1. Síndrome de Laugier-Hunziker: diagnóstico diferencial

\begin{tabular}{|c|c|}
\hline Máculas melánicas & Melanoniquia \\
\hline Síndrome Peutz-Jeghers & Onicomicosis \\
\hline Síndrome Cronkhite-Canada & Infección bacteriana \\
\hline Neurofibromatosis tipo 1 & Hematoma subungueal \\
\hline Síndrome McCune-Albright & Melanoniquia fisiológica racial \\
\hline Enfermedad de Addison & Traumatismo \\
\hline Complejo de Carney & Inducida por drogas \\
\hline Sindrome LEOPARD & Nevus \\
\hline Inducida por drogas & Melanoma \\
\hline Melanosis del fumador & Enfermedad de Bowen \\
\hline Melanoma & Pigmentación exógena \\
\hline Nevus melanocitico intraoral & Asociada a liquen plano \\
\hline
\end{tabular}

Síndrome de Cronkhite-Canada ${ }^{12}$, Neurofibormatosis tipo 1, hiperpigmentación inducida por drogas; incluidas en este grupo tetraciclinas, antimaláricos (cloroquina e hidroxicloroquina), antifúngicos (ketoconazol), quimioterápicos (ciclofosfamida, doxorrubicina, hidroxiurea), amiodarona, psicotrópicos (clorpromazina) y anticonceptivos orales, las máculas melánicas consecuentes al uso de tabaco, pigmentación causada por infección bacteriana, onicomicosis, melanoniquia traumática 0 melanoma (Tabla 1).

En casos confirmados de SLH es importante asegurar al paciente la naturaleza benigna de las lesiones y la importancia de las medidas de protección solar para evitar el empeoramiento y recurrencia de estas. El tratamiento, que es meramente cosmético, debe considerarse sólo en los casos graves con compromiso estético y/o afectación psicológica. En la actualidad, existen múltiples opciones disponibles que incluyen láser de neodimio, itrio y aluminio (láser Nd-YAG), láser Alexandrita Q-switched. Se han descrito casos de tratamiento efectivo con criocirugía.

\section{BIBLIOGRAFÍA}

1. Duan N, Zhang YH, Wang WM, Wang X. Mystery behind labial and oral melanotic macules: Clinical, dermoscopic and pathological aspects of Laugier-Hunziker syndrome. World J Clin Cases. 2018;6(10):322-334.

2. Veraldi S, Cavicchini S, Benelli C, Gasparini G. Laugier-Hunziker syndrome: a clinical, histopathologic, and ultrastructural study of four cases and review of the literature. J Am Acad Dermatol. 1991;25(4):632-636.

3. Lampe AK, Hampton PJ, Woodford-Richens K, Tomlinson I, Lawrence CM, Douglas FS. Laugier-Hunziker syndrome: an important differential diagnosis for PeutzJeghers syndrome. J Med Genet. 2003;40(6):e77.

4. Laugier P, Hunziker N. Pigmentation mélanique lenticulaire, essentielle, de la muqueuse jugale et des lèvres [Essential lenticular melanic pigmentation of the lip and cheek mucosa]. Arch Belg Dermatol Syphiligr. 1970;26(3):391-399.

5. Baran R. Longitudinal melanotic streaks as a clue to Laugier-Hunziker syndrome. Arch Dermatol. 1979;115(12):1448-1449.

6. Moore RT, Chae KA, Rhodes AR. Laugier and Hunziker pigmentation: a lentiginous proliferation of melanocytes. J Am Acad Dermatol. 2004;50(5 Suppl):S70-S74.

7. Gencoglan G, GerceKer-Turk B, Kilinc-Karaarslan I, Akalin T, Ozdemir F. Dermoscopic findings in Laugier-Hunziker syndrome. Arch Dermatol. 2007;143: 631-3.

8. Nikitakis NG, Koumaki D. Laugier-Hunziker syndrome: case report and review of the literature. Oral Surg Oral Med Oral Pathol Oral Radiol. 2013;116(1):e52-e58.

9. Sachdeva S, Sachdeva S, Kapoor P. Laugier-hunziker syndrome: a rare cause of oral and acral pigmentation. J Cutan Aesthet Surg. 2011;4(1):58-60.

10. Collins MT, Singer FR, Eugster E. McCune-Albright syndrome and the extraskeletal manifestations of fibrous dysplasia. Orphanet J Rare Dis. 2012;7 Suppl 1(Suppl 1):S4.

11. McCarthy PM, Piehler JM, Schaff HV, et al. The significance of multiple, recurrent, and "complex" cardiac myxomas. J Thorac Cardiovasc Surg. 1986;91(3):389-396

12. Cronkhite LW Jr, Canada WJ. Generalized gastrointestinal polyposis; an unusual syndrome of polyposis, pigmentation, alopecia and onychotrophia. N Engl J Med. 1955:252(24):1011-1015 